\title{
Involvement of OsGF14b Adaptation in the Drought Resistance of Rice Plants
}

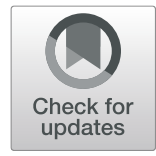

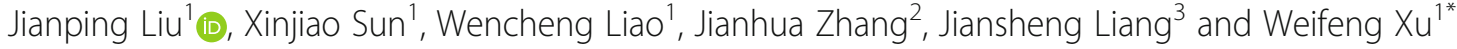

\begin{abstract}
Background: Drought stress is one of the major abiotic stresses that restrict plant growth and development. 14-33 proteins have been validated to regulate many biological processes in plants. Previous research demonstrated that OsGF14b plays different roles in panicle and leaf blast resistance. In this study, we researched the function of OsGF14b in drought resistance in rice.

Findings: Here, we report that OsGF14b was strongly induced by soil drought stress. In comparison with wild type (WT), the osgfi4b mutant exhibited improved resistance to drought and osmotic stress by changing the content of stress-relevant parameters, complementation of the osgfi4b mutant restored the drought sensitivity to WT levels, whereas the OsGF14b-overexpression lines exhibited enhanced sensitivity to drought and osmotic stress. The osgf14b mutant plants were hypersensitive to abscisic acid (ABA), while the OsGF14b-overexpression plants showed reduced sensitivity to ABA. Furthermore, mutation and overexpression of OsGF14b affected the expression of stress-related genes under normal growth conditions and/or drought stress conditions.

Conclusions: We have demonstrated that OsGF14b is involved in the drought resistance of rice plants, partially in an ABA-dependent manner.
\end{abstract}

Keywords: ABA, Rice, 14-3-3, Drought resistance, OsGF14b

\section{Findings}

Drought is one of the main abiotic stresses affecting plant growth and yield. Sessile plants have evolved various effective mechanisms to cope with drought stress $(\mathrm{Hu}$ and Xiong 2014). Obtaining a better understanding of the molecular and genetic mechanism by which plants respond to drought stress has been the subject of intensive research over the past decade, and is expected to provide and essential foundation for future breeding and genetic engineering strategies (Xiang et al. 2008; Marshall et al. 2012; Tang et al. 2016; Srivastava et al. 2017; Liang et al. 2018; Lee et al. 2018; Yao et al., 2018).

14-3-3 proteins mainly function through binding and modulating the function of phosphorylated client proteins (de Boer et al. 2013). These are localized to various subcellular compartments and regulate a wide range of cellular

\footnotetext{
* Correspondence: wfxu@fafu.edu.cn

${ }^{1}$ Center for Plant Water-use and Nutrition Regulation and College of Life Sciences, Joint International Research Laboratory of Water and Nutrient in Crop, Fujian Agriculture and Forestry University, Jinshan, Fuzhou 350002, China

Full list of author information is available at the end of the article
}

processes (Paul et al. 2012). In higher plants, 14-3-3 proteins comprise a protein family and play important roles in regulating plant development and stress responses (Comparot et al. 2003; Denison et al. 2011). Some studies have implicated the function of 14-3-3 s in drought resistance from Arabidopsis, maize and Glycine soja (He et al. 2015; Campo et al. 2012; Sun et al. 2014). In rice, at least eight 14-3-3 isoforms (OsGF14 a-h) have been identified, and these isoforms display different expression patterns under various biotic and abiotic stresses (Chen et al. 2006; $\mathrm{Xu}$ and Shi 2006; Yashvardhini et al. 2018). The different roles of OsGF14e and OsGF14b in disease resistance have been reported (Manosalva et al. 2011; Liu et al. 2016b; Liu et al. 2016a). However, only OsGF14c's roles in drought resistance were confirmed (Ho et al. 2013), and the functions of the other rice 14-3-3 proteins in this process are still unknown.

Chen et al. (2006) reported that OsGF14b was rapidly induced at $2-4 \mathrm{~h}$ by the PEG6000 (drought-mimic). To further confirm this, we applied quantitative real-time PCR (qRT-PCR) to examine the expression of OsGF14b under prolonged soil drought treatment (withholding 
water) at the 4-leaf stage. It was found that OsGF14b was strongly induced at $1 \mathrm{~d}$ (2.3-fold) and $2 \mathrm{~d}$ (3-fold), and then the transcripts returned to the pre-treatment level at $3 \mathrm{~d}$ and $4 \mathrm{~d}$, which finally remained at a higher level (2.3-fold) after recovery (Additional file 1: Figure S1). According to the above results, we speculated that OsGF14b may play a positive role in regulating drought resistance. We firstly searched for RISD (Rice T-DNA Insertion Sequence Database) and purchased the heterozygous mutant 2D-00086, a transfer DNA (T-DNA) insertion line in the japonica rice DongJin (DJ) background. The T-DNA was inserted into the promoter of OsGF14b, $745 \mathrm{bp}$ upstream of the translation initiation site (ATG), and the homozygous mutant named osgf $14 b$ was identified by PCR analysis (Additional file 1: Figure S2 and Additional file 2: Table S1). The expression level of OsGF14b in the mutant was dramatically repressed (Fig. 1a). For drought stress, the osgf $14 b$ mutant and DJ seedlings (5.5- to 6.5-leaf stage) were transplanted to plastic basins with a mixture of sand and soil (1:1), and then the irrigation was withheld for $12 \mathrm{~d}$, followed by rewatering for $7 \mathrm{~d}$ (Additional file 3). Contrary to our prediction, the osgf $14 b$ mutant showed increased resistance to drought stress. After recovery, about $56.3 \%$ of the osgf14b mutant seedlings survived compared with $12.5 \%$ of DJ seedlings (Fig. 1b). Importantly, complementation of the osgf $14 b$ mutant by introducing OsGF14b coding sequence under control of $35 \mathrm{~S}$ promoter restored the drought sensitivity to DJ levels, thereby providing compelling proof that loss of OsGF14b function is causative for the observed drought-resistant mutant phenotype (Fig. 1a, b). To further validate the function of OsGF14b in drought stress response, we requested and obtained two independent OsGF14b-overexpression (OE) lines (OE-2 and OE-4) based on the background of Nipponbare (Nip), in which the OsGF14b gene was driven by $35 \mathrm{~S}$ promoter and its expression level was significantly increased (Fig. 1a). Under normal growth conditions, we did not observe any phenotypic differences between these two overexpression lines and Nip. Under the drought stress treatment (without water for $8 \mathrm{~d}$ and recovered for $7 \mathrm{~d}$ ), both OE- 2 and OE-4 became more sensitive than Nip (Fig. 1c). After recovery, the survival rates of the OsGF14b-OE lines $(40.0 \%-52.5 \%)$ were significantly lower than that of Nip (75\%). Together, these observations supported that OsGF14b may act as a negative regulator in drought resistance.

Stomatal status is generally important for drought response in plants, so we measured the stomatal conductance of the WT and transgenic plants (mutant and OE) under normal and drought conditions at 5.5- to 6.5-leaf stage. Under normal conditions, the stomatal conductance of $o s g f 14 b$ was significantly higher than that of DJ, whereas the stomatal conductance of OsGF14b-OE lines was significantly lower than that of Nip; Under drought conditions (without water for $3 \mathrm{~d}$ ), the stomatal conductance of all plants was decreased compared with under normal conditions, but there was no significant difference between the WT and transgenic plants (Additional file 1: Figure S3). The results showed that although OsGF14b could negatively regulate the stomatal conductance under normal conditions, but had almost no impact on that under drought conditions, and stomatal conductance may be not associated with the drought resistance negatively regulated by OsGF14b.

Stresses usually cause damage in plants via oxidative stress involving the generation of reactive oxygen species (ROS), such as hydrogen peroxide $\left(\mathrm{H}_{2} \mathrm{O}_{2}\right)$ ( $\mathrm{Zhu} 2001$ ). Malondialdehyde (MDA) is an indicator of oxidative attack on membrane lipids and ion leakage reflects membrane injury (Ouyang et al. 2010). Thus, we tested the $\mathrm{H}_{2} \mathrm{O}_{2}$ and MDA content of the leaves from transgenic (mutant or OE) and WT plants (DJ or Nip). After drought stress, the $\mathrm{H}_{2} \mathrm{O}_{2}$ and MDA content in the osgf14b mutant were all less than DJ, whereas the OsGF14b-OE lines accumulated more $\mathrm{H}_{2} \mathrm{O}_{2}$ and MDA than Nip. Under normal conditions, we found that these two OsGF14b-OE lines had fewer $\mathrm{H}_{2} \mathrm{O}_{2}$ than Nip, and OE-4 had higher MDA than Nip, but there were no significant differences on these between osgf14b and DJ (Fig.1d, e). Proline and soluble sugar are two important solutes in plant cells for improving drought resistance by increasing osmotic pressure (Zhou et al. 2009). Furthermore, we also examined the content of proline and soluble sugar. After drought stress, compared with WT, the proline levels of the osgf14b mutant increased, but those of OsGF14b-OE lines declined. All tested plants had similar proline content under normal growth conditions (Fig. 1f). When the soluble sugar content was compared, the osgf14b mutant showed higher soluble sugar levels than DJ, while the OsGF14b-OE lines showed lower sugar levels than Nip under both normal and drought conditions (Fig. 1g). Taken together, these results suggested that OsGF14b may negatively regulate the resistance to drought stress via changing the content of stress-relevant parameters.

In order to investigate if OsGF14b functions under osmotic stress, we first sowed the surface-sterilized seeds on normal 1/2 MS medium and 10\% PEG4000 supplemented-1/2 MS medium. During the germination, we calculated the germination rate at different times (1 d, 2 d, 3 d, 4 d, 5 d, 6 d and 7 d). Under normal conditions, there was no difference on the germination rate among all the genotypes, and all the genotypes started to germinate at $2 \mathrm{~d}$; under the conditions of PEG4000, all the genotypes started to germinate at $3 \mathrm{~d}$. From $3 \mathrm{~d}$ to 5 $\mathrm{d}$, the germination rate of osgf $14 \mathrm{~b}$ was always higher than DJ, but the germination rate of OsGF14b-OE lines was always lower than Nip. Notably, the degree of 


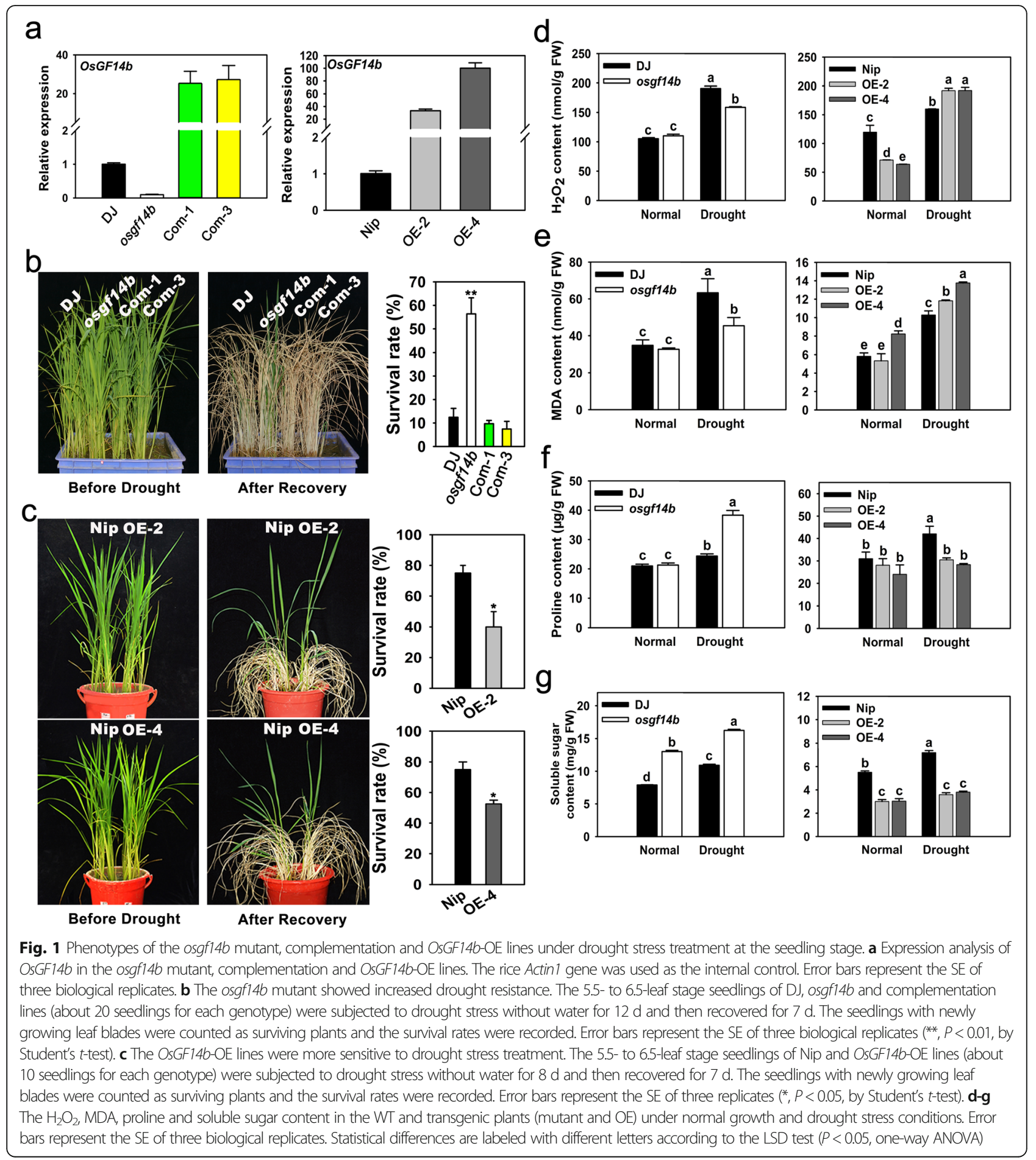

difference on germination rate between osgf $14 b$ and DJ was obviously higher than that between OsGF14b-OE lines and Nip (Additional file 1: Figure S4). Accordingly, at germination level, the drought response in the mutant may be earlier and greater, and the drought response in OsGF14b-OE lines may be later and weaker. In addition, after germination on normal $1 / 2$ MS medium, the transgenic plants together with WT control were subjected to $200 \mathrm{mM}$ mannitol treatment. When grown in normal medium, there were no obvious distinctions on shoot length between the transgenic and WT plants. When grown in mannitol-supplemented medium, the osgf $14 b$ mutant had significantly longer shoot than DJ, whereas the OsGF14b-OE lines had shorter shoot than 
Nip (Fig. 2a, b, c). The results demonstrated that OsGF14b also play a negative role in osmotic resistance, which was consistent with its role in drought resistance.

Abscisic acid (ABA) signaling plays major roles in the drought stress (Zhang et al. 2006; Tang et al. 2016), and two previous studies showed that OsGF14b could be strongly induced by ABA (Chen et al. 2006; Yao et al. 2007). So we tested if OsGF14b is involved in ABA sensitivity of rice, which is an important aspect of ABA-dependent regulation. The osgf14b mutant and two OE lines (OE-2 and OE-4) were treated with $5 \mu \mathrm{M}$ ABA, together with WT control. As shown in Fig. 2d, the osgf14b mutant seedlings were more sensitive to ABA compared to DJ. Moreover, the shoot length of the osgf14b mutant was significantly shorter than that of DJ under ABA treatment, but there was no significant difference under normal conditions. On the contrary, we found that the ABA sensitivity of OsGF14b-OE seedlings was decreased compared to Nip. In addition, the shoot length of OsGF14b-OE lines was much longer than that of Nip under ABA treatment. Nevertheless, no significant difference in these phenotypes was observed under normal conditions (Fig. 2e, f). Taken together, these results indicated that OsGF14b functions as a negative regulator of $\mathrm{ABA}$ signaling.

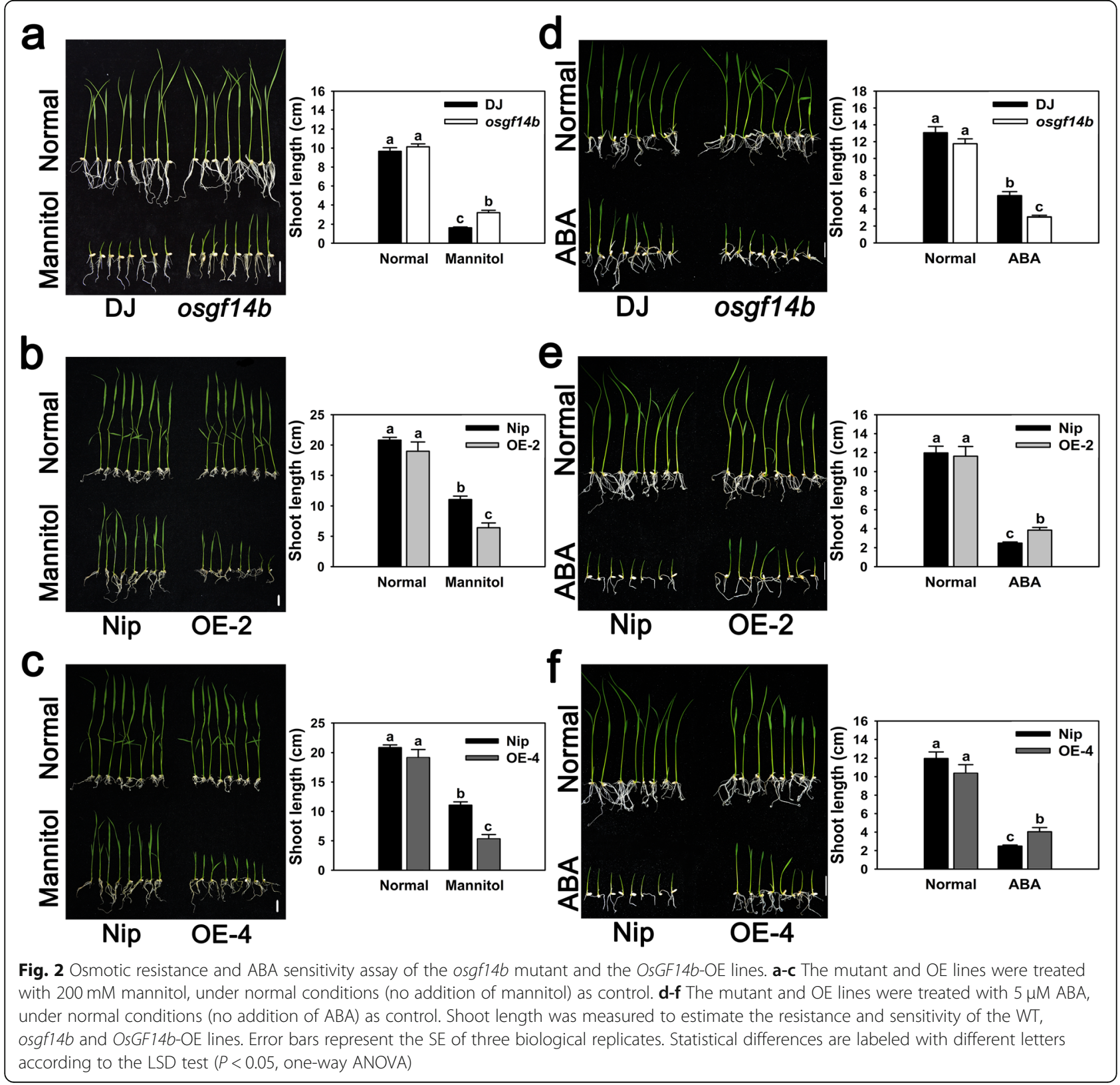



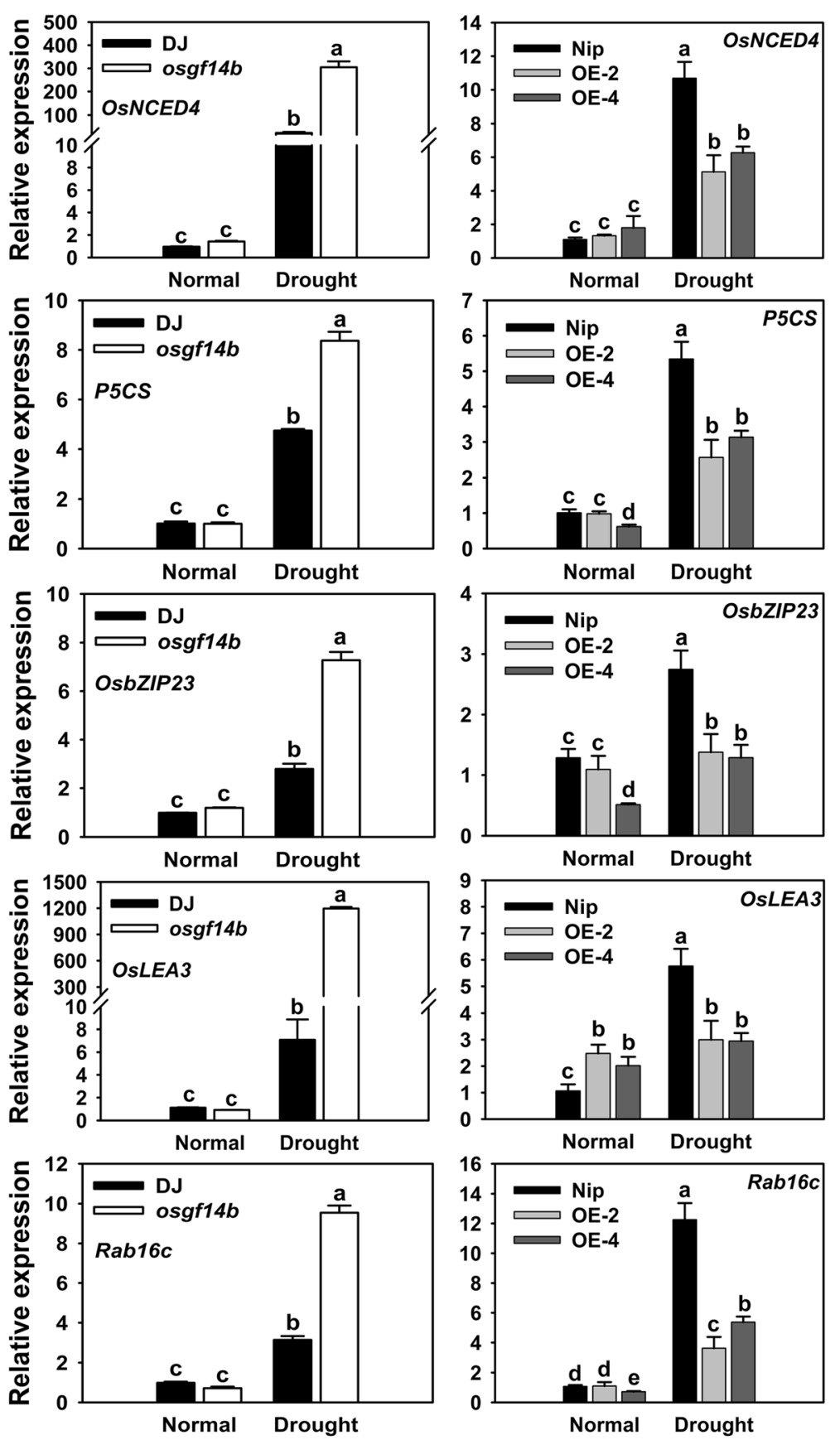

Fig. 3 The expression of abiotic stress-responsive genes in the WT and transgenic plants (mutant and OE) under normal growth and drought stress conditions. The rice Actin1 gene was used as the internal control. Error bars represent the SE of three biological replicates. Statistical differences are labeled with different letters according to the LSD test $(P<0.05$, one-way ANOVA)

To explore the possible molecular mechanisms by which OsGF14b negatively regulates drought resistance in rice, we determined the expression profiles for several well-known stress-responsive genes under normal growth and drought conditions. These included OsNCED4, encoding protein involved in ABA biosynthesis (Zhu et al. 2009); P5CS, encoding a rate-limiting enzyme involved the biosynthesis of proline (Hien et al. 2003); OsbZIP23, encoding a typical stress-related bZIP-type transcription factor (Xiang et al. 2008); OsLEA3 and Rab16c, encoding late embryogenesis abundant (LEA) proteins (Xiao et al. 2007; El-Esawi and Alayafi 2019). Our results displayed that compared to under normal conditions, the expression of OsNCED4, P5CS, OsbZIP23, OsLEA3 and Rab16c was constitutively elevated in the transgenic (mutant and OE) and WT plants under drought stress conditions. However, 
after drought stress, the expression levels of these genes were significantly higher in the osgf $14 b$ mutant than that in DJ, and lower expression levels of these genes in the OsGF14b-OE lines were observed compared with Nip (Fig. 3). These results showed that OsGF14b may negatively regulate drought resistance by altering the expression of stress-responsive genes.

In conclusion, in this study we have demonstrated that OsGF14b is involved in the rice drought and osmotic resistance via changing the contents of stress-relevant parameters and the expression of stress-related genes, partially in an ABA-dependent manner. This findings presented here will provide a novel insight into the function of OsGF14b in rice.

\section{Additional Files}

Additional file 1. Figure S1. Expression levels of OSGF14b under soil drought stress treatment. Figure S2. Schematic diagram of the OsGF14b gene and PCR-based genotyping for the osgf14b homozygous mutant. Figure S3. Stomatal conductance of the WT and transgenic plants under normal and drought conditions. Figure $\mathbf{S 4}$. Seeds germination rate of the WT and transgenic lines on normal medium and 10\% PEG4000 supplemented-medium.

Additional file 2: Table S1. List of primers used in this study (F, forward primer; $R$, reverse primer; q, quantitative RT-PCR).

Additional file 3. Materials and methods.

\section{Abbreviations}

ABA: Abscisic acid; CDS: Coding sequence; d: day; DJ: DongJin; $\mathrm{H}_{2} \mathrm{O}_{2}$ : Hydrogen peroxide; LSD: Least significant difference; MDA: Malondialdehyde; MS: Murashige and skoog; Nip: Nipponbare; OE: Overexpression; PEG: Polyethylene glycol; qRT-PCR: Quantitative real time polymerase chain reaction; RISD DB: Rice T-DNA Insertion Sequence Database; ROS: Reactive oxygen species; WT: Wild type

\section{Acknowledgements}

The authors would like to thank Prof. Bin Liu (Rice Research Institute, Guangdong Academy of Agricultural Sciences, Guangzhou, China) for providing the seeds of OsGF14b-overexpression lines.

\section{Authors' Contributions}

LJP and XWF contributed to the experimental design. LJP, SXJ and LWC contributed to experiment performance and data analysis. LJP and XWF drafted the manuscript. ZJH and LJS contributed to good advice on designing the experiment and revising the manuscript. All authors read and approved the final manuscript.

\section{Funding}

This study was supported by the National Natural Science Foundation of China (31601232), Fujian Agriculture and Forestry University Program for Distinguished Young Scholar (xja201706), the Natural Science Foundation of Fujian Province (2017 J05046), and China Postdoctoral Science Foundation (2017 M612108)

\section{Availability of Data and Materials}

All data supporting the conclusions of this article are provided within the article (and its additional files).

\section{Ethics Approval and Consent to Participate}

Not applicable.

\section{Consent for Publication}

Not applicable.

\section{Competing Interests}

The authors declare that they have no competing interests.

\section{Author details}

${ }^{1}$ Center for Plant Water-use and Nutrition Regulation and College of Life Sciences, Joint International Research Laboratory of Water and Nutrient in Crop, Fujian Agriculture and Forestry University, Jinshan, Fuzhou 350002, China. ${ }^{2}$ Department of Biology, Hong Kong Baptist University, Hong Kong, China. ${ }^{3}$ Department of Biology, Southern University of Science and Technology, Shenzhen 518055, China.

Received: 27 July 2019 Accepted: 4 November 2019

Published online: 14 November 2019

\section{References}

Campo S, Peris-Peris C, Montesinos L, Penas G, Messeguer J, Segundo BS (2012) Expression of the maize ZmGF14-6 gene in rice confers tolerance to drought stress while enhancing susceptibility to pathogen infection. J Exp Bot 63: 983-999

Chen F, Li Q, Sun L, He Z (2006) The rice 14-3-3 gene family and its involvement in responses to biotic and abiotic stress. DNA Res 13:53-63

Comparot S, Lingiah G, Martin T (2003) Function and specificity of 14-3-3 proteins in the regulation of carbohydrate and nitrogen metabolism. J Exp Bot 54:595-604

de Boer AH, van Kleeff PJM, Gao J (2013) Plant 14-3-3 proteins as spiders in a web of phosphorylation. Protoplasma 250:425-440

Denison FC, Paul AL, Zupanska AK, Ferl RJ (2011) 14-3-3 proteins in plant physiology. Semin Cell Dev Biol 22:720-727

El-Esawi MA, Alayafi AA (2019) Overexpression of Rice Rab7 gene improves drought and heat tolerance and increases grain yield in Rice (Oryza sativa L.). Genes (Basel) 10:56

He YC, Wu JJ, Lv B, Li J, Gao ZP, Xu WF, Baluska F, Shi WM, Shaw PC, Zhang JH (2015) Involvement of 14-3-3 protein GRF9 in root growth and response under polyethylene glycol-induced water stress. J Exp Bot 66:2271-2281

Hien DT, Jacobs M, Angenon G, Hermans C, Thu TT, Son LV, Roosens NH (2003) Proline accumulation and $\Delta^{1}$-pyrroline-5-carboxylate synthetase gene properties in three rice cultivars differing in salinity and drought tolerance. Plant Sci 165:1059-1068

Ho SL, Huang LF, Lu CA, He SL, Wang CC, Yu SP, Chen J, Yu SM (2013) Sugar starvation- and GA-inducible calcium-dependent protein kinase 1 feedback regulates GA biosynthesis and activates a 14-3-3 protein to confer drought tolerance in rice seedlings. Plant Mol Biol 81:347-361

Hu H, Xiong L (2014) Genetic engineering and breeding of drought-resistant crops. Annu Rev Plant Biol 65:715-741

Lee H, Cha J, Choi C, Choi N, Ji HS, Park SR, Lee S, Hwang DJ (2018) Rice WRKY11 plays a role in pathogen defense and drought tolerance. Rice 11:5

Liang J, Guo S, Sun B, Liu Q, Chen X, Peng H, Zhang Z, Xie Q (2018) Constitutive expression of REL1 confers the rice response to drought stress and abscisic acid. Rice 11:59

Liu Q, Yang J, Zhang S, Zhao J, Feng A, Yang T, Wang X, Mao X, Dong J, Zhu X, Leung H, Leach JE, Liu B (2016a) OsGF14b positively regulates panicle blast resistance but negatively regulates leaf blast resistance in Rice. Mol PlantMicrobe Interact 29:46-56

Liu Q, Yang J, Zhang S, Zhao J, Feng A, Yang T, Wang X, Mao X, Dong J, Zhu X, Leung H, Leach JE, Liu B (2016b) OsGF14e positively regulates panicle blast resistance in rice. Biochem Biophys Res Commun 471:247-252

Manosalva PM, Bruce M, Leach JE (2011) Rice 14-3-3 protein (GF14e) negatively affects cell death and disease resistance. Plant J 68:777-787

Marshall A, Aalen RB, Audenaert D, Beeckman T, Broadley MR, Butenko MA, CanoDelgado Al, de Vries S, Dresselhaus T, Felix G, Graham NS, Foulkes J, Granier C, Greb T, Grossniklaus U, Hammond JP, Heidstra R, Hodgman C, Hothorn M, Inze D, Ostergaard L, Russinova E, Simon R, Skirycz A, Stahl Y, Zipfel C, De Smet I (2012) Tackling drought stress: receptor-like kinases present new approaches. Plant Cell 24:2262-2278

Ouyang SQ, Liu YF, Liu P, Lei G, He SJ, Ma B, Zhang WK, Zhang JS, Chen SY (2010) Receptor-like kinase OsSIK1 improves drought and salt stress tolerance in rice (Oryza sativa) plants. Plant J 62:316-329

Paul AL, Denison FC, Schultz ER, Zupanska AK, Ferl RJ (2012) 14-3-3 Phosphoprotein interaction networks-does isoform diversity present functional interaction specification? Front Plant Sci 3:190 
Srivastava AK, Zhang C, Caine RS, Gray J, Sadanandom A (2017) Rice SUMO protease overly tolerant to salt 1 targets the transcription factor, OsbZIP23 to promote drought tolerance in rice. Plant J 92:1031-1043

Sun XL, Luo X, Sun MZ, Chen C, Ding XD, Wang XD, Yang SS, Yu QY, Jia BW, Ji W, Cai H, Zhu YM (2014) A Glycine Soja 14-3-3 protein GsGF140 participates in Stomatal and root hair development and drought tolerance in Arabidopsis thaliana. Plant Cell Physiol 55:99-118

Tang N, Ma SQ, Zong W, Yang N, Lv Y, Yan C, Guo ZL, Li J, Li X, Xiang Y, Song HZ, Xiao JH, Li XH, Xiong LZ (2016) MODD mediates deactivation and degradation of OsbZIP46 to negatively regulate ABA signaling and drought resistance in Rice. Plant Cell 28:2161-2177

Xiang Y, Tang N, Du H, Ye HY, Xiong LZ (2008) Characterization of OsbZIP23 as a key player of the basic Leucine zipper transcription factor family for conferring Abscisic acid sensitivity and salinity and drought tolerance in Rice. Plant Physiol 148:1938-1952

Xiao B, Huang Y, Tang N, Xiong L (2007) Over-expression of a LEA gene in rice improves drought resistance under the field conditions. Theor Appl Genet 115:35-46

Xu WF, Shi WM (2006) Expression profiling of the 14-3-3 gene family in response to salt stress and potassium and iron deficiencies in young tomato (Solanum lycopersicum) roots: analysis by real-time RT-PCR. Ann Bot 98:965-974

Yao LY, Cheng X, Gu ZY, Huang W, Li S, Wang LB, Wang YF, Xu P, Ma H, Ge XC (2018) The AWPM-19 Family Protein OsPM1 Mediates Abscisic Acid Influx and Drought Response in Rice. Plant Cell 30: 1258-1276

Yao Y, Du Y, Jiang L, Liu JY (2007) Molecular analysis and expression patterns of the 14-3-3 gene family from Oryza sativa. J Biochem Mol Biol 40:349-357

Yashvardhini N, Bhattacharya S, Chaudhuri S, Sengupta DN (2018) Molecular characterization of the 14-3-3 gene family in rice and its expression studies under abiotic stress. Planta 247:229-253

Zhang JH, Jia WS, Yang JC, Ismail AM (2006) Role of ABA in integrating plant responses to drought and salt stresses. Field Crop Res 97:111-119

Zhou W, Li Y, Zhao BC, Ge RC, Shen YZ, Wang G, Huang ZJ (2009) Overexpression of TaSTRG gene improves salt and drought tolerance in rice. J Plant Physiol 166:1660-1671

Zhu GH, Ye NH, Zhang JH (2009) Glucose-induced delay of seed germination in Rice is mediated by the suppression of ABA catabolism rather than an enhancement of ABA biosynthesis. Plant Cell Physiol 50:644-651

Zhu JK (2001) Plant salt tolerance. Trends Plant Sci 6:66-72

\section{Publisher's Note}

Springer Nature remains neutral with regard to jurisdictional claims in published maps and institutional affiliations.

\section{Submit your manuscript to a SpringerOpen ${ }^{\circ}$ journal and benefit from:}

- Convenient online submission

- Rigorous peer review

- Open access: articles freely available online

- High visibility within the field

- Retaining the copyright to your article

Submit your next manuscript at $\boldsymbol{\nabla}$ springeropen.com 